\title{
The Effects of Inverse Ratio Ventilation with PEEP on Respiratory Function and Inflammatory Cytokines in Patients during One-lung Ventilation
}

\section{Zhang WP $^{1^{*}}$ and Zhu SM${ }^{2}$}

${ }^{1}$ Department of Anesthesiology, Jiaxing Maternity and Child Health Care Hospital, Shanghai, China

${ }^{2}$ Department of Anesthesiology, The First Affiliated Hospital of Medical School, Zhejiang University, Zhejiang Province, China

\begin{abstract}
Background: Hypoxemia is the most common complication during one-lung ventilation (OLV). Inverse ratio ventilation (IRV) may lead to intrinsic positive end-expiratory pressure (PEEP) and improve oxygenation in acute respiratory distress syndrome, so we investigated whether volume-controlled IRV with external PEEP could improve hypoxemia, reduce the risk of acute lung injury during OLV.

Methods: Sixty patients undergoing one-lung ventilation for open thoracoctomy were randomly divided into IRV group and control group $(n=30)$. All patients were initially ventilated with a tidal volume of $8 \mathrm{~mL} / \mathrm{kg}$, an inspiratory: expiratory (I:E) ratio of $1: 2$ and a respiratory rate of 12 breaths/min in $100 \%$ oxygen without PEEP. During OLV, lungs were ventilated either with I:E of 2:1 (IRV group) or 1:2 (control group) with an actual tidal volume (VT) $7 \mathrm{~mL} / \mathrm{kg}$, respiratory rate 12 breaths/min, external PEEP of $5 \mathrm{~cm} \mathrm{H}_{2} \mathrm{O}$. Arterial blood was collected respectively to analyze blood gas before and during OLV. Meanwhile, hemodynamic and respiratory mechanics were monitored. The concentrations of IL-1 $\beta$, IL- 6 and IL-8 in bronchoalveolar lavage fluid (BALF) were measured before and during OLV.

Results: Compared to the control group, partial pressure of arterial oxygen $\left(\mathrm{PaO}_{2}\right)$, mean airway pressure and dynamic compliance $(C L)$ were significantly higher in IRV group during OLV $(P<0.05)$. However, plateau pressure $\left(P_{\text {plat }}\right)$ and levels of IL-1 $\beta$, IL-6 and IL-8 in BALF were lower in IRV group than those in control group $(P<0.05)$.

Conclusion: IRV (I:E = 2:1) applying PEEP could improve hypoxemia, promote oxygenation, and improve dynamic compliance of respiratory system, moreover reduce $\mathrm{P}_{\text {tat }}$ and the release of inflammatory cytokines in patients during one-lung ventilation. It is superior to conventional ventilation with PEEP during one-lung ventilation.
\end{abstract}

Keywords: Inverse ratio ventilation; One-lung ventilation; Hypoxemia; Lung injury

\section{Introduction}

The incidence of hypoxemia is reportedly from $5 \%$ to $10 \%$ during one-lung ventilation (OLV) [1]. High tidal volume and airway pressure during OLV correlated with the development of acute ventilationinduced lung injury in patients undergoing lung resection [2]. Inverse ratio ventilation (IRV) has been proposed for patients with adult respiratory distress syndrome to achieve adequate oxygenation $[3,4]$, due to recruiting atelectatic alveoli $[5,6]$, and it is reported that the optimal inspiratory to expiratory ratio (I:E) is 2:1 when using IRV [7]. It is widely accepted the use of low tidal volume should be accompanied by positive end-expiratory pressure (PEEP), and the previous study reported that PEEP could improve oxygenation $[8,9]$, but it was rarely reported that IRV (I:E $=2: 1$ ) applying external PEEP was used in thoracic surgery. The present study tested the hypothesis that IRV (I:E = 2:1) applying external PEEP would improve hypoxemia, promote oxygenation and alleviate inflammation in patients underwent one-lung ventilation for lung lobectomy, is superior to conventional ventilation with PEEP. The primary endpoint was that IRV (I:E = 2:1) applying external PEEP would reduced $\mathrm{P}_{\text {plat }}$

\section{Materials and Methods}

This study was approved by the Hospital Ethics Committee of Jiaxing maternity and child health care hospital and registered in protocol registration system (registration number: ChiCTR-IOR-15006517). The informed consent was signed before enrollment by the patients. From May 2015 to December 2015, we identified 60 patients undergoing elective lung lobectomy with ASA grade II, age 34-61 years, body mass index (BMI) $21-29 \mathrm{~kg} / \mathrm{m}^{2}$ and minute ventilation volume $>70 \mathrm{~L} / \mathrm{min}$ to enroll in this study. We excluded the patients that had the history of severe cardiovascular disease, asthma, chronic obstructive pulmonary disease and pneumothorax. There were 38 central type lung cancers and 22 peripheral type lung cancers among. The duration of the operation lasted 86.4-138.2 min. Sixty patients were randomly assigned to either control group $(\mathrm{n}=30)$ or IRV group $(\mathrm{n}=30)$ using computer-generated random number code.

Upon arrival in the operating room, standard monitoring including electrocardiogram, invasive arterial blood pressure (ABP), heart rate (HR), pulse oximetry $\left(\mathrm{SpO}_{2}\right)$ and central venous pressure (CVP) were applied and venous access was established. Anesthesia was induced with intravenous propofol $2 \mathrm{mg} / \mathrm{kg}$, fentanyl $5 \mu \mathrm{g} / \mathrm{kg}$ and rocuronium $0.6 \mathrm{mg} /$ $\mathrm{kg}$, then bronchial intubation was performed using direct laryngoscope. Anesthesia was maintained with propofol 6-10 mg. $\mathrm{kg}^{-1} \cdot \mathrm{h}^{-1}$ and remifentanil 0.5-1 ug. $\mathrm{kg}^{-1} \cdot \mathrm{min}^{-1}$ to keep the bispectrail index (BIS) value between 40 to 55 (BIS monitor Model A2000, USA). The lungs were mechanically ventilated with ventilator (DATAX-OHMEDA Aspire anesthesia machine, USA). Muscle relaxation was monitored by the train-of-four (TOF) stimulation on the ulnar nerve (Organon, Type TOF-Watch SX, Holland). Continuous infusion of rocuronium was performed to

*Corresponding author: Zhang WP, Department of Anesthesiology, Jiaxing Maternity and Child Health Care Hospital, Zhonghuan East Road, Jiaxing City, Zhejiang Province, China, Tel: +8613757370679; E-mail: zhang650679@163.com

Received February 25, 2016; Accepted March 23, 2016; Published March 28, 2016

Citation: Zhang WP, Zhu SM (2016) The Effects of Inverse Ratio Ventilation with PEEP on Respiratory Function and Inflammatory Cytokines in Patients during Onelung Ventilation. J Pulm Respir Med 6: 329. doi:10.4172/2161-105X.1000329

Copyright: @ 2016 Zhang WP, et al. This is an open-access article distributed under the terms of the Creative Commons Attribution License, which permits unrestricted use, distribution, and reproduction in any medium, provided the original author and source are credited. 
maintain stable neuromuscular block.

The trachea was intubated using a double-lumen tube (37F for males and $39 \mathrm{~F}$ for females), the correct position of the double-lumen tube was confirmed using a fiber-optic bronchoscope (Olympus company, Tokyo, Japan) after intubation in both supine and lateral positions. All patients were initially applied with two-lung ventilation. The lungs were initially ventilated using a constant-flow, volume-controlled ventilation mode with an actual tidal volume of $8 \mathrm{~mL} / \mathrm{kg}$ actual body weight, an inspiratory to expiratory (I:E) ratio of $1: 2$, a respiratory rate of 12 breaths/min, oxygen flow rate of $1 \mathrm{~L} / \mathrm{min}$, and $\mathrm{FiO}_{2}$ (fraction of inspiratory oxygen) of 1.0 without PEEP. OLV was initiated at the moment of skin incision, and the tube lumen of the non-ventilated lung was opened to room air. During OLV, tidal volume was reduced to $7 \mathrm{~mL} / \mathrm{kg}$, and ventilator settings were actual tidal volume $\left(\mathrm{V}_{\mathrm{T}}\right)$ of 7

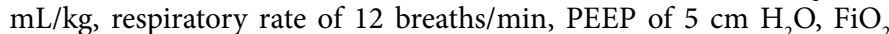
(fraction of inspiratory oxygen) of 1.0 , oxygen flow rate of $1 \mathrm{~L} / \mathrm{min}, \mathrm{I}: \mathrm{E}$ of 2:1 (in IRV group) or 1:2 (in control group). At the time of closure of thoracic cavity, two-lung ventilation was started and both lungs were re-expanded by hand bagging in all patients.

Mean airway pressure $\left(\mathrm{P}_{\text {mean }}\right)$, plateau airway pressure $\left(\mathrm{P}_{\text {plat }}\right)$, total PEEP $\left(\mathrm{PEEP}_{\text {tot }}\right)$, dynamic compliance of respiratory system (CL) and end-tidal $\mathrm{CO}_{2}$ partial pressure $\left(\mathrm{PetCO}_{2}\right)$ were monitored using a sidestream spirometry device (Anesthesia Monitor D-FPD15-00; GE, Taipei, Taiwan), maintaining $\mathrm{PetCO}_{2}$ value of $35-45 \mathrm{mmHg}$. When $\mathrm{PetCO}_{2}>45 \mathrm{mmHg}$, tidal volume or breath rate was increased. All the patients were in lateral position with head down $10^{\circ}$ during surgery. If $\mathrm{SpO}_{2}$ was below $92 \%$ and lasted 30 seconds during OLV, surgery was temporarily interrupted and double-lung ventilation was alternately applied. The neuromuscular block was reversed with intravenous neostigmine $1 \mathrm{mg}$ and atropine $0.5 \mathrm{mg}$ when TOF $>70 \%$. Fentanyl 1 $\mu \mathrm{g} / \mathrm{kg}$ was administered for pain control at $30 \mathrm{~min}$ before the end of surgery. Postoperative complications were observed, such as time in Post Anesthesia Care Unit (PACU), time to extubation and incidence of hypoxemia. The Patient was discharged from PACU when the modified Aldrete score was appropriate (score $\geq 9$ ) [10]. Patients were followed up for any complications of lung during their hospital stays. Throughout the perioperative operation, lactated Ringer's solution or hydroxyethyl starch solution was infused at rate of $8-10 \mathrm{ml} \cdot \mathrm{kg}^{-1} \cdot \mathrm{h}^{-1}$.

Hemodynamic parameters, such as systolic blood pressure (SBP), diastolic blood pressure (DBP), HR and CVP were recorded at $5 \mathrm{~min}$ before anesthesia induction (T0), $5 \mathrm{~min}$ after bronchial intubation (T1), the initiate of OLV (T2), 45 min of OLV (T3) and the end of surgery (T4), meanwhile, $\mathrm{P}_{\text {mean }}, \mathrm{P}_{\mathrm{plat}}, \mathrm{PEEP} \mathrm{P}_{\text {tot }}$ and CL were recorded at T1, T2, T3 and T4. Arterial blood gas and mixed venous blood gas were analyzed using a blood gas analyzer (ABL8000A, Denmark) and bronchoalveolar lavage fluid (BALF) of ventilated lung at $\mathrm{T} 1$ and $\mathrm{T} 3$ point. At $\mathrm{T} 1$ and $\mathrm{T} 3$ point, we collected bronchoalveolar lavage fluid performed by infilliting $50 \mathrm{~mL}$ normal saline in ventilated lung and $20-40 \%$ this fluid was recovered with fiber bronchoscope (Olympus company, Tokyo, Japan), then samples were centrifuged at $3,000 \mathrm{rpm}$ for 15 minutes at $4^{\circ} \mathrm{C}$, saved for assay at $-70^{\circ} \mathrm{C}$. IL- $1 \beta$, IL- 6 and IL- 8 levels were detected by enzuyme-linked immunosorbent assay (ELISA), using the microplate reader (Hyperion MR III, USA). All enzyme-linked immunosorbent assays were performed according to the manufacturers' guidelines.

\section{Statistical analysis}

Descriptive statics and compliance with normal distribution was examined with one-sample Kolmogorov-Smirnov test. Between groups Student $t$ test and Mann-Whitney $U$-test were used. Categorical variables were evaluated with the Chi-square test. Data were presented as mean \pm standard deviation. Parametric data were checked for by using $t$ test or categorical data was analyzed by Chi-square test. Significance was considered as $P$ value $<0.05$. Data analysis was performed using the statistical software package SPSS 19.0 (SPSS Inc, Chicago, USA). Sample size based on a previous trial test. The main variable in the study, $\mathrm{P}_{\text {plat }}$ was having a standard deviation of 2.1 from a plot test done on 10 patients. A priori power analysis using two-sided analysis with an a-error of 0.05 and a power of 0.8 showed that 54 patients were needed for this study.

\section{Results}

No significant differences in age, body mass index, gender, maximal ventilation volume (MMV), time of OLV and operation time between groups (Table 1) $(P>0.05)$. At T3, $\mathrm{PaO}_{2}$ increased significantly in IRV group, there was statistical significance between groups (Table 2) ( $P$ $<0.01)$. Comparison of $\mathrm{pH}, \mathrm{PaCO}_{2}, \mathrm{SaO}_{2}$ and $\mathrm{ScvO}_{2}$, there were no statistical significance in both groups $(P>0.05)$.

The $\mathrm{P}_{\text {mean }}, \mathrm{PEEP}_{\text {tot }}$ and CL were increased significantly in IRV group than those in control group at T1, T2, T3 and T4 (Table 3), there was statistically significant between groups $(P<0.05)$, but $\mathrm{P}_{\text {plat }}$ was lower in IRV group (Figure 1), there was statistical significance $(P<0.05)$. Hemodynamic monitoring reflects the hemodynamic changes in both groups (Figure 2). The comparison of hemodynamic parameters in both groups, there was no statistical significance $(P>0.05)$. The concentrations of IL- 6 , IL- 8 and IL- $1 \beta$ were lower in IRV group than those in the control group at T3, there was statistically significant between the groups $(P<0.05)$ (Table 3$)$. Hypoxemia was 1 case in IRV group and 3 cases in control group during OLV (Table 1), however there was statistical significance between two groups $(P<0.05)$. There was no significance in the incidence of intraoperative hypoxemia and time in PACU between two groups $(P>0.05)$ (Table 1$)$. No postoperative hypoxemia and hypoxemia was observed.

\section{Discussion}

One-lung ventilation is a common technique of ventilation in thoracic surgery, which is advantageous to surgical area because of the non-ventilated lung collapse, prevents from the secretions into the healthy lung and protects the healthy lung from pollution. Meanwhile the collapse lung will increase the intrapulmonary shunt, aggravate hypoxia and increase airway pressure of the ventilated lung to result in acute lung injury. High tidal volume and plateau pressure are the major risk factor of acute lung injury, thus the present study compared volume-controlled inverse ratio ventilation ( $\mathrm{I}: \mathrm{E}=2: 1$ ) with PEEP to conventional volume-controlled ventilation ( $\mathrm{I}: \mathrm{E}=1: 2)$ with PEEP in patients undergoing one-lung ventilation.

Lower $\mathrm{P}_{\text {plat }}$, higher $\mathrm{P}_{\text {mean }}$, total PEEP and CL were observed in IRV group compared with the control group. Lower $\mathrm{P}_{\text {plat }}$ in IRV group was possibly because of the longer inspiratory time or slow inspiratory flow, and higher $\mathrm{P}_{\text {mean }}$ was achieved by moderate prolongation of I:E ratio [11]. Prolonging inspiratory time resulted in increases in $\mathrm{P}_{\text {mean }}$ and decreases in $\mathrm{P}_{\text {peak }}$ or $\mathrm{P}_{\text {plat }}$ in IRV group. Therefore, when applying low external PEEP, IRV is considered to be superior to conventional ratio ventilation in terms of gas exchange and respiratory mechanics in this study.

The mechanisms of improving oxygenation applying IRV were possibly as following: Firstly, $\mathrm{P}_{\text {mean }}$ is thought to be a major determinant because it is correlated with mean alveolar pressure and alveolar recruitment [11]. Secondly, increased inspiratory time may have enough time to gas exchange effectively. Lastly, PEEP was an important 
Citation: Zhang WP, Zhu SM (2016) The Effects of Inverse Ratio Ventilation with PEEP on Respiratory Function and Inflammatory Cytokines in Patients during One-lung Ventilation. J Pulm Respir Med 6: 329. doi:10.4172/2161-105X.1000329

Page 3 of 6

\begin{tabular}{|c|c|c|c|}
\hline Index & IRV & Control & $P$ value \\
\hline Age (year) & $56.0 \pm 13.1$ & $54.3 \pm 12.7$ & 0.5198 \\
\hline $\mathrm{BMI}\left(\mathrm{kg} / \mathrm{cm}^{2}\right)$ & $24.0 \pm 3.2$ & $23.4 \pm 2.8$ & 0.7254 \\
\hline Gender (male/female) & $17 / 13$ & $16 / 14$ & 0.0671 \\
\hline MMV (L/min) & $85.2 \pm 11.8$ & $87.4 \pm 13.2$ & 0.5014 \\
\hline Duration of OLV (min) & $68.9 \pm 12.6$ & $73.1 \pm 16.1$ & 0.2694 \\
\hline Duration of operation (min) & $116.5 \pm 26.8$ & $123.2 \pm 31.6$ & 0.3828 \\
\hline PACU discharge time (min) & $57.2 \pm 14.6$ & $53.3 \pm 13.2$ & 0.2865 \\
\hline Intra-operative hypoxemia & 1 & 3 & 0.7894 \\
\hline
\end{tabular}

Data are mean \pm SD or number. BMI: Body Mass Index; MMV: Maximal Ventilation Volume; PACU: Post Anesthesia Care Unit.

Table 1: Data of patients in both groups.

\begin{tabular}{|c|c|c|c|}
\hline & IRV & Control & $P$ value \\
\hline \multicolumn{4}{|c|}{ Arterial blood gas at $\mathrm{T} 1$ point } \\
\hline $\mathrm{pH}$ & $7.37 \pm 0.04$ & $7.36 \pm 0.02$ & 0.1676 \\
\hline $\mathrm{PaO}_{2}(\mathrm{mmHg})$ & $89.5 \pm 7.8$ & $91.6 \pm 8.7$ & 0.1994 \\
\hline $\mathrm{PaCO}_{2}(\mathrm{mmHg})$ & $35.2 \pm 2.8$ & $34.5 \pm 2.5$ & 0.2476 \\
\hline $\mathrm{SaO}_{2}(\%)$ & $99.82 \pm 0.13$ & $99.79 \pm 0.17$ & 0.2749 \\
\hline $\mathrm{ScvO}_{2}(\%)$ & $68.7 \pm 2.6$ & $69.4 \pm 2.8$ & 0.2974 \\
\hline \multicolumn{4}{|c|}{ Arterial blood gas at T3 point } \\
\hline $\mathrm{pH}$ & $7.32 \pm 0.06$ & $7.34 \pm 0.07$ & 0.1802 \\
\hline $\mathrm{PaO}_{2}(\mathrm{mmHg})$ & $232.7 \pm 45.6$ & $196.5 \pm 35.5$ & $0.0043^{*}$ \\
\hline $\mathrm{PaCO}_{2}(\mathrm{mmHg})$ & $38.65 \pm 5.4$ & $36.87 \pm 3.9$ & 0.2407 \\
\hline $\mathrm{SaO}_{2}(\%)$ & $99.68 \pm 1.8$ & $99.56 \pm 1.6$ & 0.7549 \\
\hline $\mathrm{ScvO}_{2}(\%)$ & $77.9 \pm 3.1$ & $78.3 \pm 3.4$ & 0.7962 \\
\hline
\end{tabular}

Data are mean $\pm S D$, *Means statistically significant $(p<0.05)$. T1: At $5 \mathrm{~min}$ after bronchial intubation; T3: At 45 min of one-lung ventilation; ScvO : Oxygen saturation of central venous blood.

Table 2: Arterial blood gas in both groups.

\begin{tabular}{|l|l|l|l|}
\hline \multicolumn{2}{|l|}{ IRV } & Control & P value \\
\hline At T1 point & & & \\
\hline IL-1 $\beta(\mathrm{mp} / \mathrm{L})$ & $76.5 \pm 14.5$ & $72.8 \pm 12.4$ & 0.2967 \\
\hline IL-6 $(\mathrm{mp} / \mathrm{L})$ & $118.6 \pm 30.6$ & $112.4 \pm 23.1$ & 0.3828 \\
\hline IL-8 $(\mathrm{mp} / \mathrm{L})$ & $83.6 \pm 17.9$ & $80.4 \pm 15.8$ & 0.4686 \\
\hline At T3 point & & & \\
\hline IL-1 $\beta(\mathrm{mp} / \mathrm{L})$ & $91.6 \pm 21.2$ & $106.7 \pm 26.7$ & $0.0215^{*}$ \\
\hline IL-6 $(\mathrm{mp} / \mathrm{L})$ & $149.3 \pm 34.6$ & $177.8 \pm 40.3$ & $0.0063^{*}$ \\
\hline IL-8 $(\mathrm{mp} / \mathrm{L})$ & $102.4 \pm 22.4$ & $117.7 \pm 25.6$ & $0.0197^{*}$ \\
\hline
\end{tabular}

Data are mean \pm standard deviation. Compared with the control group, *Means statistically significant $(p<0.05)$. T1: At the initiate of mechanical ventilation; T3: At 45 min of one-lung ventilation; IL: Interleukin.

Table 3: The levels of IL $-1 \beta$, IL-6 and IL-8 in both groups ( $\mathrm{mp} / \mathrm{L})$.

factor probably to improve oxygenation, which facilitated the mixing of gas, made collapse alveoli reopen and prevented atelectasis.

IRV would increase the $\mathrm{P}_{\text {mean }}$, recruit atelectatic alveoli, reduce intrapulmonary shunt, improve ventilation-perfusion mismatch and decrease dead space ventilation [12]. Prolonged inspiratory time allows enough time to gas exchange. Meanwhile, as expiratory time was shorter, inverse ratio ventilation might lead to air trapping in the lungs with generation of auto-PEEP (internal PEEP). PEEP could increase the mean airway pressure and improve oxygenation. Documents are proved that IRV may lead to intrinsic PEEP [13] and is thought to improve oxygenation and to have advantageous effects on lung mechanics [14]. Higher mean airway pressure was benefit to gas exchange and prevented from lung collapse. $\mathrm{PaCO}_{2}$ was showed modest decrease in IRV group compared to the control group, there was no significance between two groups. IRV didn't affect elimination of $\mathrm{CO}_{2}$ [15], which was agreement with the present study that IRV with PEEP didn't prolong the time to

extubation and increase the postoperative pulmonary complications.

In our study, there was no significant difference on hemodynamic parameters between two groups, such as: SBP, DBP, HR and CVP. It was suggested that IRV (I:E = 1:2) applying PEEP didn't hamper venous return with possible consequent hemodynamic derangement. It is consistent with the previous statement that increasing the percentage of inspiratory time had no demonstrable changes in hemodynamic during mechanical ventilation [16].

In this experiment, the IL- $1 \beta$, IL- 6 and IL- 8 of BALF were increased significantly at $45 \mathrm{~min}$ of one-lung ventilation in both groups, and levels of IL- $1 \beta$, IL- 6 and IL-8 in IRV group were significantly lower compared with the control group. One-lung ventilation caused inflammatory response possibly because inflammation factors were activated and released. Inflammatory factors play an important role in the stress response, including IL-1 $\beta$, IL- 6 and IL- 8 , which are important proinflammatory factor [17]. In the mechanical lung injury, IL-6 is one of the most important inflammatory mediators, its concentration was positively correlated with the degree of lung injury [18]. Neutrophils play a central role in the inflammatory response. Upon infection and/ or tissue damage, the neutrophils must first adhere to endothelium in response to chemokines presented at the endothelial interface and then migrate out of the microvasculature by following other chemokines secreted from nearby macrophages, mast cells, and other serosal cells. The neutrophils would then follow a series of end target chemoattractants such as IL-8 or LTB4 to the final site of infection [19]. In addition, damaged-molecular-patterns (DAMPs) are molecules that have a physiological role inside the cell, but acquire additional functions when they are exposed to the extracellular environment: they alert the body about danger, stimulate an inflammatory response, and 

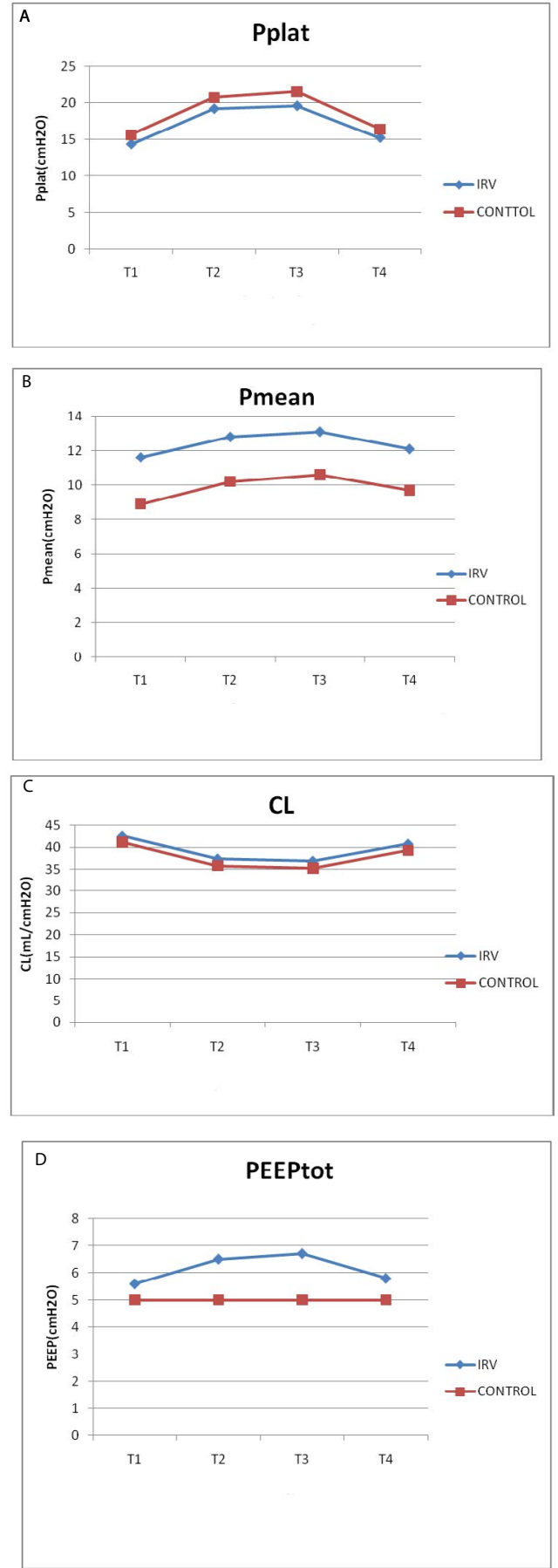

Figure 1: Comparison of respiratory parameters of patients in both groups. A) Comparison of Pplat at different time in two groups (P < 0.001$)$. $X$ axis-time point, $Y$ axis-plateau pressures. B) Comparison of mean airway pressures different time $(P<0.001)$. $X$ axis-time point, $Y$ axis-mean airway pressure. $C)$ Comparison of $C L$ at different time in two groups $(P<0.05)$. $X$ axis-time point, $Y$ axis-dynamic compliance. $D)$ Comparison of total $P E E P$ at different time $(P<0.05)$. $X$ axis-time point, Y axis-total PEEP. T0: At 5 min before anesthesia induction; T1: At 5 min after bronchial intubation; T2: At initiate of OLV; T3: At 45 min after OLV; T4: The end of surgery.

finally promote the regeneration process. Beside their passive release by dead cells, some DAMPs can be secreted or exposed by living cells undergoing a life-threatening stress [20]. DAMPs could target the cytokines synthesis during hypoxic condition. They played a role in the inflammatory response, so that the release of the IL- $1 \beta$, IL- 6 and
IL- 8 would increase in lung tissue. The IL-1 $\beta$, IL- 6 and IL- 8 were large released on the condition of hypoxia or lung tension. Studies suggested that inverse ratio ventilation could alleviate the inflammatory response.

The typical forms of lung injury are as following: volutrauma, barotraumas, atelectrauma and biological lung injury [21]. In this 
Citation: Zhang WP, Zhu SM (2016) The Effects of Inverse Ratio Ventilation with PEEP on Respiratory Function and Inflammatory Cytokines in Patients during One-lung Ventilation. J Pulm Respir Med 6: 329. doi:10.4172/2161-105X.1000329
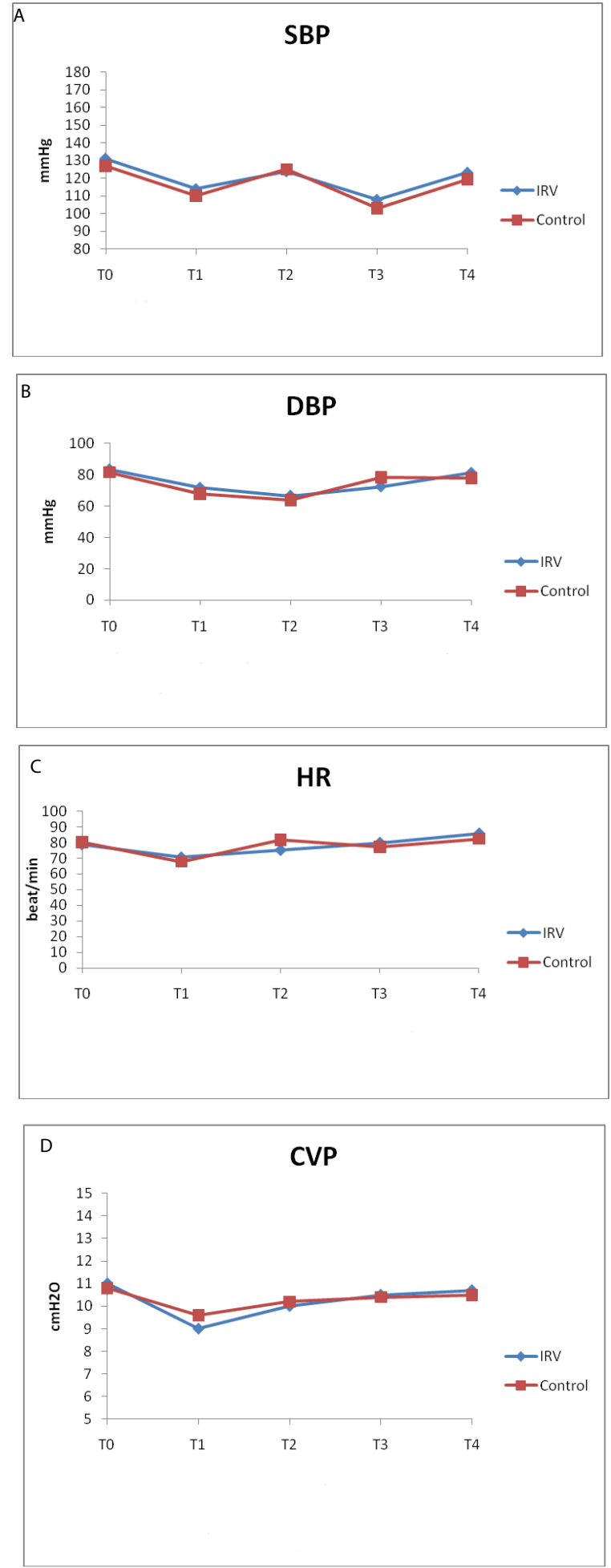

Figure 2: Comparison of hemodynamic parameters in both groups. A) Comparison of systolic pressure in two groups ( $p>0.05$ ), $X$ axis-time, $Y$ axis-systolic pressure in $\mathrm{mmHg}$. B) Comparison of diastolic blood pressure in two groups ( $p>0.05), X$ axis-time, $Y$ axis- diastolic blood pressure. C) Comparison of diastolic heart rates in two groups ( $p>0.05$ ), $X$ axis-time, $Y$ axis- heart rate. D) Comparison of CVP in two groups ( $p>0.05)$, X axis-time, $Y$ CVP. T0: At 5 min before anesthesia induction; T1: At 5 min after bronchial intubation; T2: At initiate of OLV; T3: At 45 min after OLV; T4: The end of surgery. 
Citation: Zhang WP, Zhu SM (2016) The Effects of Inverse Ratio Ventilation with PEEP on Respiratory Function and Inflammatory Cytokines in Patients during One-lung Ventilation. J Pulm Respir Med 6: 329. doi:10.4172/2161-105X.1000329

study, some degree of inflammation was already present probably due to surgery, trauma, anesthesia and injurious mechanical ventilation, and so on. Inverse ratio ventilation could low the $\mathrm{P}_{\text {plat }}$ increase $\mathrm{P}_{\text {mean }}$ and alleviate the inflammatory response. It maybe alleviate acute lung injury. Using inverse ratio ventilation, we obtained good ventilation and prevented lung injury with PEEP. It is consistent with the protective ventilation strategy.

IRV is different from the normal ratio ventilation: there may be some shortcomings or potential risks, whether will cause respiratory dysfunction or other adverse effects remain to be studied further. Moreover, the other limitation of this study is that anesthesiologist is not blinded to ventilation strategy.

To sum up, IRV applying PEEP can improve hypoxemia, promote oxygenation and increase dynamic compliance of respiratory system. Therefore, when applying low external PEEP, IRV is considered to be superior to conventional ratio ventilation in terms of gas exchange and respiratory mechanics in patients during OLV.

\section{Acknowledgement}

We are indebted to Dr. Wang LZ for providing editorial review for manuscript preparation.

\section{Authorship and Contributorship}

Zhang WP collected the data and performed the research and Zhu SM performed the research.

\section{Ethics}

The study was approved by the Ethics Committee of Hospital.

\section{Conflict of Interest}

There are no conflicts of interest in connection with this article.

\section{References}

1. Karzai W, Schwarzkopf K (2009) Hypoxemia during one-lung ventilation: prediction, prevention, and treatment. Anesthesiology 110: 1402-1411.

2. Fernández-Pérez ER, Keegan MT, Brown DR, Hubmayr RD, Gajic O (2006) Intraoperative tidal volume as a risk factor for respiratory failure after pneumonectomy. Anesthesiology 105: 14-18.

3. Mercat A, Titiriga M, Anguel N, Richard C, Teboul JL (1997) Inverse ratio ventilation $(I / E=2 / 1)$ in acute respiratory distress syndrome: a six-hour controlled study. Am J Respir Crit Care Med 155: 1637-1642.

4. Huang CC, Shih MJ, Tsai YH, Chang YC, Tsao TCY, et al. (2001) Effects of inverse ratio ventilation versus positive end-expiratory pressure on gas exchange and gastric intramucosal $\mathrm{PaCO}(2)$ and $\mathrm{pH}$ under constant mean airway pressure in acute respiratory distress syndrome. Anesthesiology 95 $1182-1188$

5. Gurevitch MJ, Van Dyke J, Young ES, Jackson K (1986) Improved oxygenation and lower peak airway pressure in severe adult respiratory distress syndrome: Treatment with inverse ratio ventilation. Chest 89: 211

6. Talab HF, Zabani IA, Abdelrahman HS, Bukhari WL, Mamoun I, et al. (2009) Intraoperative ventilatory strategies for prevention of pulmonary atelectasis in obese patients undergoing laparoscopic bariatric surgery. Anesth Analg 109: $1511-1516$.

7. Sari A, Yamashita S, Toriumi T, Nakashima K, Kawata R, et al. (1991) The effects of inversed ratio ventilation (IRV) on arterial oxygenation during mechanical ventilation in patients with acute respiratory failure. Resuscitation 22: 93-101.

8. Maracaja-Neto LF, Vercosa N, Roncally AC, Giannella A, Bozza FA, et al. (2009) Beneficial effects of high positive end-expiratory pressure in lung respiratory mechanics during laparoscopic surgery. Acta Anaesthesiol Scand 53: 210-207.

9. Karsten J, Luepschen H, Grossherr M, Bruch HP, Leonhardt S, et al. (2011) Effect of PEEP on regional ventilation during laparoscopic surgery monitored by electrical impedance tomography. Acta Anaesthesiol Scand 55: 878-886.

10. Aldrete JA (1995) The post-anesthesia recovery score revisited. J Clin Anesth 7: 89-91.

11. Pinhu L, Whitehead T, Evans T, Griffiths M (2003) Ventilator-associated lung injury. Lancet 361: 332-340.

12. Pesenti A, Marcolin R, Prato P, Borelli M, Riboni A, et al. (1985) Mean airway pressure vs. positive end-expiratory pressure during mechanical ventilation. Crit Care Med 13: 34-37.

13. Andersson LE, Baath M, Thorne A, Aspelin P, Odeberg-Wernerman S (2005) Effect of carbon dioxide pneumoperitoneum on development of atelectasis during anesthesia, examined by spiral computed tomography. Anesthesiology 102: 293-299.

14. Ludwigs U, Klingstedt C, Baehrendtz S, Hedenstierna G (1997) A comparison of pressure- and volume-controlled ventilation at different inspiratory to expiratory ratios. Acta Anaesthesiol Scand 41: 71-77.

15. Sinha M, Chiplonkar S, Ghanshani R (2012) Pressure-controlled inverse ratio ventilation using laryngeal mask airway in gynecological laparoscopy. J Anaesthesiol Clin Pharmacol 28: 330-333.

16. Manthous CA, Lewis TH (1993) Increased blood pressure during inverse ratio ventilation in two patients with adult respiratory distress syndrome. Chest 104 $1625-1627$.

17. Slutsky AS, Imai Y (2003) Ventilator-induced lung injury, cytokines, PEEP, and mortality: implications for practice and for clinical trials. Intensive Care Med 29: 1218-1221.

18. Boutten A, Dehoux MS, Seta N, Ostinelli J, Venembre P, et al. (1996) Compartmentalized IL-8 and elastase release within the human lung in unilateral pneumonia. Am J Respir Crit Care Med 153: 336-342.

19. Heit B, Tavener S, Raharjo E, Kubes $P$ (2002) An intracellular signaling hierarchy determines direction of migration in opposing chemotactic gradients. J Cell Biol 159: 91-102.

20. Vénéreau E, Ceriotti C, Bianchi ME (2015) DAMPs from Cell Death to New Life. Front Immunol 6: 422.

21. Manicone AM (2009) Role of the pulmonary epithelium and inflammatory signals in acute lung injury. Expert Rev Clin Immunol 5: 63-75. 